\title{
AVALIAÇÃO DA LONGITUDINALIDADE NA ATENÇÃO PRIMÁRIA EM SAÚDE PARA USUÁRIOS NO MUNICÍPIO DE SANTA ROSA/RS
}

\section{EVALUATION OF LONGITUDINALITY IN PRIMARY HEALTH CARE FOR USERS IN THE MUNICIPALITY OF SANTA ROSA/RS}

\author{
Julia da Rosa Tolazzi \\ Especialista em Gestão Pública \\ Universidade Federal de Santa Maria \\ juliadarosa@hotmail.com \\ Luis Felipe Dias Lopes \\ Doutor em Engenharia de Produção e Sistemas \\ Universidade Federal de Santa Maria \\ flopes67@yahoo.com.br \\ Nuvea Kuhn \\ Mestra em Desenvolvimento e Políticas Públicas \\ Universidade Federal de Santa Maria \\ nuvea.kuhn@iffarroupilha.edu.br \\ Claudia Aline de Souza Ramser \\ Mestra em Engenharia da Produção \\ Universidade Federal de Santa Maria \\ claudiaramser@gmail.com
}

\begin{abstract}
RESUMO
A longitudinalidade enquanto um dos atributos da Atenção Primária em Saúde constitui um vínculo temporal existente entre usuário e o sistema de saúde. Trata-se de um importante indicador para o sistema de saúde no país. Para fins de atentar a este atributo a nível local em um município localizado na região noroeste do estado do Rio Grande do Sul, no presente estudo tem-se como objetivo avaliar a longitudinalidade do cuidado pela perspectiva dos usuários no município de Santa Rosa/RS. Para tal, utilizou-se de um estudo transversal, de caráter quantitativo e descritivo-analítico, desenvolvido no período de março de 2018 com 100\% ( $n=17)$ das Equipes de Saúde da Família do município. Participaram do estudo 200 usuários adultos, por meio da aplicação do instrumento Primary Care Assessment Tool com 14 itens. O estudo permitiu identificar a insatisfação dos usuários quanto à longitudinalidade no serviço de saúde no município. Diante de tal realidade, ressalta-se a importância da reestruturação dos serviços de saúde, juntamente com a sensibilização dos profissionais que atuam neste intuito e que estão em contato próximo com o paciente, com o objetivo de fortalecer o vínculo com o usuário e melhorar o desempenho nas ações realizadas rotineiramente nas unidades de saúde.
\end{abstract}

Palavras-chave: Atenção Primária em Saúde. Estratégia Saúde da Família. Longitudinalidade.

\begin{abstract}
Longitudinality as one of the attributes of Primary Health Care constitutes a temporal link that exists between the user and the health system. It is an important indicator for the health system in the country. In order to pay attention to this attribute at the local level in a city located in the northwest region of the state of Rio Grande do Sul, this study aims to assess the longitudinality of care from the perspective of users in the city of Santa Rosa/RS. To this end, we used a cross-sectional study, with a quantitative and descriptive-analytical character, developed in the period of March 2018 with $100 \% \quad(n=17)$ of the Family Health Teams in the municipality. 200 adult users participated in the study, through the application of the 14-item Primary Care Assessment Tool. The study allowed us to identify the users'
\end{abstract}

Recebido em: 04/02/2020

Aceito para publicação em: 19/07/2021. 
dissatisfaction regarding the longitudinality of the health service in the city. In view of this reality, the importance of restructuring health services is highlighted, together with the awareness of professionals who work for this purpose and who are in close contact with the patient, in order to strengthen the bond with the user and improve the performance in actions routinely performed in health units.

Keywords: Primary Health Care. Family Health Strategy. Longitudinality.

\section{INTRODUÇÃO}

A Atenção Primária à Saúde (APS) é considerada o primeiro nível de assistência no sistema de saúde (HARZHEIM et al., 2016, ALVES, 2020, SAPS, 2020). Caracteriza-se por uma atenção ambulatorial não especializada que opera por meio de unidades de saúde, e pelo desenvolvimento de um conjunto diversificado de atividades clínicas de baixa densidade tecnológica (LAVRAS, 2011). No Brasil, a APS é considerada a "menina dos olhos" do Sistema Único de Saúde (SUS), por meio da Estratégia Saúde na Família (ESF) (ARAÚJO et al., 2011, SAPS, 2020).

A longitudinalidade pode ser caracterizada pela atenção orientada ao usuário. Tem como atributo principal o acompanhamento, a conexão interpessoal, e a credibilidade realizada ao longo do tempo entre o profissional de saúde e o paciente (STARFIELD, 2002). Na prestação de serviços em saúde, pode ser considerada uma relação de vínculo entre usuário e equipe de saúde (HARMUCH; BARATIERI, 2017). Esse vínculo longitudinal (VL) pode ser considerado "um atributo essencial da APS, com um papel fundamental no processo de trabalho em saúde da família e no cuidado" (SANTOS; ROMANO; ENGSTROM, 2018, p.1).

Visando a reestruturação da APS, a longitudinalidade tem como característica principal a melhoria na saúde pública e no empenho dos cuidados clínicos (GÉRVAS; FERNÁNDEZ; SÁNCHEZ, 2012). Por esse motivo, é reconhecida como característica principal da APS, sendo almejada para todos os participantes que participam de seus processos (CUNHA; GIOVANELLA, 2011).

Ao longo de mais de 30 de atuação do SUS, houve um significativo avanço na assistência à saúde em diversas regiões brasileiras, considerando-se principalmente como pontos positivos a queda de mortalidade e a diminuição de doenças transmissíveis (SOUZA et al., 2018). No entanto, os desafios de atuação do SUS ainda são muitos, e que incluem sua atuação frente a novas doenças (SOUZA et al., 2018), aumento da expectativa de vida populacional (SZWARCWALD, 2017; SCHENKER; COSTA, 2019), entre outros fatores que merecem atenção no que tange à assistência primária em saúde, tais como a dificuldade de acesso ao serviço por parte dos usuários e a própria sobrecarga de atendimentos pelos profissionais ao realizarem as visitas periódicas nos âmbitos familiares (SCHENKER; COSTA, 2019).

Diante tais aspectos, e da relevância de pesquisas voltadas à saúde pública no país, no presente estudo tem-se como objetivo avaliar a longitudinalidade do cuidado pela perspectiva dos usuários no município de Santa Rosa/RS. Trata-se de um município considerado referência em atendimento e saúde na região noroeste no atendimento em várias especialidades, e que se destaca na busca pela constante padronização dos procedimentos e serviços (FUMSSAR, 2018).

Santa Rosa desenvolve um importante papel e suporte no atendimento à saúde também às demandas dos demais municípios que compõem a região noroeste (CASTRO; ANES; DALCIN, 2019). Junto a mais 21 municípios da região, o município faz parte da14a Coordenadoria Regional de Saúde (CRS) (SECRETARIA DA SAÚDE, 2021). Assim, este estudo apresenta sua relevância à medida que mensura um importante indicador, a longitudinalidade pela perspectiva dos usuários no município em estudo, e que a partir deste diagnóstico pode direcionar a novas práticas, estratégias e serviços por partes dos gestores, profissionais envolvidos e sistema de saúde local. Contribui não só pelo viés social, como também com o intuito de direcionar a melhorias e avanços nos serviços em saúde da região. 


\section{REFERENCIAL TEÓRICO}

Neste tópico será contextualizado sobre a atenção primária em saúde e sobre a longitudinalidade para usuários.

\section{ATENÇÃO PRIMÁRIA EM SAÚDE}

A APS pode ser conceituada por seus quatro atributos essenciais: o acesso de primeiro contato, longitudinalidade, integralidade e coordenação da assistência (STARFIELD, 2002, MINISTÉRIO DA SAÚDE, 2019), e por seus três atributos derivados: a atenção centrada na família, orientação comunitária e competência cultural dos profissionais. Por meio desse conceito, é possível identificar o grau de orientação à APS, com a associação entre a existência dos atributos e a eficiência da atenção (HARZHEIM et al., 2006, OLIVEIRA; PEREIRA, 2013). Por meio das estratégias da APS têm-se a perspectiva de uma abrangência mais ampla e resultados positivos no que diz respeito à saúde da população (TESSER; NORMAN; VIDAL, 2018).

Apesar dos avanços voltados à saúde populacional brasileira, no ano de 2019 surgiu a necessidade de uma reforma nas bases norteadoras da APS, principalmente no que tange a lacunas que são evidenciadas na prática entre a relação entre usuários do sistema de saúde e os profissionais que prestam o atendimento (HARZHEIM et al., 2020).

No SUS, a exemplo do que ocorre em sistemas de saúde em outros países no mundo, apresenta-se no quadro atual como um sistema fragmentado, que dificulta o acesso, gera descontinuidade assistencial e compromete a integralidade da atenção ofertada. Ou seja, não abrange adequadamente às necessidades inerentes à saúde populacional (LAVRAS, 2011, SILVEIRA; JORGE; LEITÃO, 2017, SOUZA et al., 2019).

Diante da incapacidade de atender as demandas por serviços de saúde, o fortalecimento do setor privado em detrimento do interesse público e do SUS, apresenta-se essencial. No entanto, neste segmento de atuação pública, existe um reflexo de produção de desigualdades sociais, e que desfavorece grupos vulneráveis da população brasileira que carecem de atendimento à saúde e assistência (SOUZA et al., 2019).

A pesquisa realizada por Rasia et al. (2020), com 50 gestores de Unidades Básicas de Saúde (UBS) no município de Pelotas/RS em 2015, demonstrou na prática as fragilidades enfrentadas no atendimento da APS. Em destaque apontaram principalmente para a demora na realização dos exames e consultas médicas. Já a pesquisa realizada por Cabral et al. (2019), em uma análise com 781 municípios de Minas Gerais/MG apontou a ineficiência no atendimento da APS. Demonstrou que sozinha a APS não consegue resolver os problemas de saúde populacional.

Dutra e Gomes (2020) atentaram a avaliação da qualidade da saúde no Brasil, e analisaram que dos 17 indicadores estabelecidos, composto por indicadores de entrada, processos e saídas, apenas sete atenderam aos critérios de qualidade.

As pesquisas apresentadas possibilitam analisar os desafios que são enfrentados na saúde pública brasileira, e que inclui a atuação das APS neste sentido. No entanto muitas ações e estratégias são realizadas com o cunho de melhor a relação e atendimento ao usuário.

Uma das estratégias que visam assegurar a efetividade da APS é a Estratégia Saúde da Família (ESF) (ARAÚJO et al., 2011, RODRIGUES; RAMIRES, 2015, SAPS, 2020). Criada em 1994, por meio do Programa Saúde da Família (PSF), mais tarde passou a ser denominada ESF e posteriormente adotada como reestruturação e ampliação da APS. Assim, ao longo dos anos possibilitou a ampliação de número de equipes para 0 atendimento e cobertura populacional (BRASIL, 2004).

Neste sentido, destaca-se a Portaria № 2.488, de 21 de outubro de 2011, que aprovou a Política Nacional de Atenção Básica (PNAB), em que por meio desta, no Brasil, pôde-se expandir o escopo de atuações da APS, definindo-as como porta de entrada preferencial do SUS e como parte do sistema municipal de saúde, principalmente pela busca de padronização do processo de trabalho das equipes (BRASIL, 2011).

A ESF define-se por um conjunto de ações e serviços que vão além da assistência médica, estruturando-se com base no reconhecimento das necessidades da população, apreendidas a partir do estabelecimento de vínculos entre os usuários 
dos serviços e os profissionais de saúde, em contato permanente com o território (OLIVEIRA; PEREIRA, 2013, p.159).

Silveira, Jorge e Leitão (2017), por meio de uma revisão integrativa, levando-se em consideração o recorte temporal de 2016 e 2017, descreveram que apesar de a ESF ser reconhecida como a porta de entrada para a APS, o cuidado integral à saúde ainda se faz distante para muitas pessoas.

A nível nacional, a abrangência e cobertura da ESF têm se tornando cada vez mais ampla. Para o ano de 2019 , constatou-se que "cerca de $60 \%$ dos municípios estavam cadastrados em Unidades de Saúde da Família (UFS). [...] A cobertura é superior nas áreas rurais e nas regiões Nordeste e Sul" (GIOVANELLA et al., 2021, p.2543). Ainda, trata-se de uma expansão significativa e que compõe em sua estrutura a cobertura de 150 milhões de pessoas por meio de suas 45 mil Unidades Básicas de Saúde (HARZHEIM et al., 2020, p.4).

No entanto, possibilitar a saúde a toda população ainda parece distante, sendo necessárias outras estratégias para atender a uma parcela significativa da população, por meio do fortalecimento da APS e da integração dos sistemas de saúde (DAMACENO et al., 2020).

Percebe-se desta forma, que a ESF por meio da APS contempla um importante aspecto no que tange a perspectiva de abrangência e atendimento a usuários em saúde, tanto a nível local quanto regional, e especificamente pensando-se o território nacional.

\section{A LONGITUDINALIDADE PARA USUÁRIOS}

A busca pela integralização da saúde da população brasileira, a gestão do cuidado, a continuidade nos atendimentos bem como o cuidado individual e coletivo podem ser considerados a base para a atuação da APS (SANTOS, 2018). Neste intuito destaca-se a existência de vínculos por meio do atributo de longitudinalidade (STARFIELD, 2002, HARMUCH; BARATIERI, 2017).

Originada da palavra longitudinal, a longitudinalidade é caracterizada pelo acompanhamento a grupos e/ou indivíduos da sociedade ao longo de um espaço de tempo (MINISTÉRIO DA SAÚDE, 2017). Caracteriza-se pelo vínculo dos usuários e profissionais de saúde em suas respectivas unidades de saúde. No cenário da APS, representa uma conexão de extensa continuidade das ações entre os atores envolvidos, com destaque para os intensos elos interpessoais, que possuem o objetivo de cooperação recíproca (STARFIELD, 2002). Representa uma relação de confiança entre usuário e ESF (ALVES, 2020).

A presença do atributo longitudinalidade é considerada um fator essencial para os serviços de saúde, pois possibilita gerar diagnósticos e intervenções pertinentes, além de direcionar a encaminhamentos à especialistas para a execução de procedimentos que exijam maior complexidade (GÉRVAS; FERNÁNDEZ, 2005). Ainda, aufere maior qualidade no cuidado e atendimento ao usuário sob a perspectiva de longo prazo (TESSER; NORMAN; VIDAL, 2018).

Para que exista a longitudinalidade, faz-se necessário a existência do vínculo entre equipe de saúde e usuários. Ao passar do tempo, usuários e profissionais de saúde passam a se conhecer melhor, o que possibilita qualificar o atendimento (STARFIELD, 2002, OLIVEIRA; PEREIRA, 2013).

O VL faz-se fundamental e essencial para o processo de APS voltados à Saúde da Família, principalmente por possibilitar a afinidade interpessoal, as estratégias de cuidado e as práticas de educação em saúde (SANTOS; ROMANO; ENGSTROM, 2018).

No Brasil, com o cunho de identificar a visão dos usuários quanto à longitudinalidade na APS, pesquisas têm sido realizadas em diferentes estados, a exemplo de Harzheim et al. (2016), no Rio de Janeiro/RJ, com 6.675 usuários adultos e cuidadores de crianças usuárias dos serviços em 2014, a de Harmuch e Baratieri (2017), com 379 usuários em Guarapuava/PR; e a de Kessler et al. (2019), com 1.076 usuários adultos em 32 municípios da 4ª Coordenadoria Regional de Saúde do Rio Grande do Sul/RS.

A existência da longitudinalidade apresenta-se como um elemento fundamental no êxito das ações pensadas em saúde pública (TESSER; NORMAN; VIDAL, 2018), pois permeia a perspectiva de relações estabelecidas ao longo do tempo, e que podem ser consideradas substanciais para a efetividade do cuidado e do atendimento ao usuário. Ainda, possibilita que sejam pensadas e implementadas ações de prevenção e tratamento precoce (DUNCAN et al., 2014). 
Destaca-se sua essencialidade frente as adversidades enfrentadas pelos usuários pensando-se seu contexto social e econômico. Assim, o estabelecimento e a manutenção desses vínculos por meio da longitudinalidade permitem não apenas a gestão do cuidado como também o acompanhamento clínico ao longo do tempo.

\section{MÉTODO}

Com o objetivo de avaliar a longitudinalidade do cuidado pela perspectiva dos usuários no município de Santa Rosa/RS, utilizou-se de um estudo quantitativo, transversal e descritivo-analítico (HAIR Jr.et al., 2005). O estudo foi realizado em 17 Equipes de Saúde da Família (ESFs) do município de Santa Rosa, cidade situada na região noroeste do estado do Rio Grande do Sul/RS com cerca de 72.504 habitantes (IBGE, 2017), que correspondem a totalidade de ESF.

A coleta de dados foi realizada no período de março de 2018. O tamanho da amostra seguiu a indicação de Hair Jr. et al. (2005) que sugerem um mínimo de 5 vezes mais observações que o número de variáveis a serem analisadas, sendo ainda mais adequado uma proporção de 10 para 1 , como o atributo da APS longitudinalidade apresenta 14 questões, portanto a amostra mínima será de 140 usuários. Foram questionados 200 usuários adultos que estavam na ESF no momento da coleta, com idade maior ou igual à 18 anos que estavam vinculados há pelo menos 1 (um) ano ao serviço municipal de saúde de Santa Rosa/RS, e excluídos usuários adultos que não estavam vinculados há pelo menos 1 (um) ano ao serviço municipal de saúde e que não apresentaram condições de saúde cognitiva para responder à pesquisa.

A coleta de dados foi realizada por meio da aplicação do instrumento Primary Care Assessment Tool (PCATool) versão adulto (BRASIL, 2010, CARVALHO et al., 2013), que contêm o atributo da APS longitudinalidade, além das questões sobre o perfil socioeconômico e demográfico dos participantes.

A ferramenta PCATool foi produzida e validada por Starfield e Shi (2002) nos Estados Unidos da América (EUA) na Johns Hopkins Primary Care Policy Center (PCPC). Essa ferramenta mensura a existência e a extensão dos atributos da APS para crianças, profissionais de saúde e usuários adultos e baseia-se nos atributos essenciais e derivados da APS sistematizados por Starfield (2002).

O instrumento original apresenta três versões autoaplicáveis, designada a crianças (PCATool versão Criança), para adultos (maiores de 18 anos) (PCATool versão Adulto) e para profissionais de saúde/coordenador/gerente do serviço de saúde (PCATool versão Profissionais de Saúde) (STARFIELD; SHI, 2002).

O PCATool mensura a presença e extensão dos atributos essenciais e derivados da APS, com aspectos de processo, estrutura e resultados dos serviços de saúde. Entre os atributos essenciais avaliados estão o acesso de primeiro contato do indivíduo com o sistema de saúde, longitudinalidade, integralidade, coordenação da atenção. Já os atributos derivados são a atenção à saúde centrada na família (orientação familiar), orientação comunitária e a competência cultural (BRASIL, 2010). Para adaptar a realizada brasileira, o documento foi traduzido e validado, com teste de confiabilidade, tornando-se uma ferramenta aplicável por meio de entrevistadores. Quando validado a ferramenta recebeu o nome de Instrumento de Avaliação da Atenção Primária - PCATool-Brasil (HARZHEIM et al., 2006).

As variáveis independentes analisadas neste estudo foram idade (anos), gênero (masculino e feminino), estado civil (solteiro, casado, outros (divorciado, viúvo e união estável)), ocupação (desempregado, funcionário público, autônomo, assalariado e beneficiário), escolaridade (ensino fundamental completo e incompleto, ensino médio completo e incompleto, ensino superior e analfabeto) e presença de plano de saúde privado (sim ou não). Também foram analisadas as variáveis: tempo de vinculação ao serviço municipal de saúde de Santa Rosa/RS (1 à 3 anos, 4 à 6 anos, 7 à 10 anos, mais de 10 anos), quantas vezes frequentou a ESF no último ano (1 à 3 vezes, 4 à 6 vezes, 7 à 10 vezes, mais de 10 vezes) e quantas vezes recebeu visita domiciliar de algum profissional da ESF no último ano (1 à 3 vezes, 4 à 6 vezes, 7 à 10 vezes, mais de 10 vezes).

A variável dependente analisada no estudo foi a longitudinalidade na APS, composta por 14 itens (BRASIL, 2010). As respostas seguiram uma escala do tipo Likert, com escores definidos $(4=\mathrm{com}$ certeza sim, 3 = provavelmente sim, $2=$ provavelmente não, $1=$ com certeza não e $9=$ não sei/não lembro). Os escores foram calculados pela média aritmética simples dos valores das respostas dos itens, e se o escore "não sei/não lembro" atingir $50 \%$ ou mais do total de itens do componente, devese desconsiderar o cálculo desse item para o entrevistado. Para a obtenção do escore final do 
atributo foi calculada a soma do valor dos itens, dividido pelo número de itens (14). O escore para a longitudinalidade é classificado em baixo escore $(<6,67)$ e alto escore $(\geq 6,67)$ (BRASIL, 2010).

Os dados coletados foram transferidos para o programa Microsoft Excel ${ }^{\circledR} 2016$ e após foram analisados com o software Statistical Analysis System (SAS) versão 9. Foi aplicado o coeficiente alfa de Cronbach para avaliar a confiabilidade das respostas, considerando valor igual ou superior a 0,70 (HAIR Jr.; TATHAM; BLACK, 2009). Os testes Qui-quadrado, Cluster Analisys e Analise de Correspondência utilizaram um nível de significância de $5 \%$. Este estudo foi submetido e aprovado pelo Comitê de Ética em Pesquisa da Universidade Regional do Estado do Rio Grande do Sul UNIJUÍ, sob número do CAAE: 79600517.7.0000.5350, seguindo os preceitos éticos da Resolução no. 466 de 12 de dezembro de 2012, do Conselho Nacional de Saúde que regulamenta a pesquisa envolvendo Seres Humanos. Assim, a pesquisa foi aplicada aos sujeitos que estavam cientes do objetivo do estudo e assinaram o Termo de Consentimento Livre e Esclarecido.

\section{RESULTADOS}

Considerando-se a população residente em 2019 no município de Santa Rosa, a maior parte dos munícipes concentra-se na faixa etária de 15 a 64 anos, o que corresponde respectivamente a 70,3\% do total da população de homens, e $69,3 \%$ de mulheres. Ademais, possui uma população predominantemente feminina, cuja escolaridade é ensino fundamental incompleto (45\%) e ensino médio completo e superior incompleto (25\%) (SEBRAE, 2020). Trata-se de uma população jovem, visto que $17,09 \%$ da população encontram-se na faixa de 20 a 29 anos (FUMSSAR, 2018). Em relação ao perfil socioeconômico e demográfico pertinentes a este estudo, averiguou-se que $74 \%(\mathrm{n}=$ 148) dos participantes eram do gênero feminino, $45 \%(n=90)$ tinham idade superior à 50 anos, $68 \%$ ( $n=136)$ eram casados, $37,50 \%(n=75)$ eram beneficiários, $54,50 \%(n=109)$ tinham ensino médio e a maioria dos participantes $94 \%(n=188)$ não possuíam plano de saúde privado, tal como apresentado na tabela 1.

Tabela 1 - Características socioeconômicas e demográficas dos participantes da pesquisa, Santa Rosa/RS, 2018

\begin{tabular}{|c|c|c|}
\hline & & \\
\hline Varıavel & $n$ & $\%$ \\
\hline Faixa Etária & & \\
\hline Até 30 anos & 47 & 23,50 \\
\hline 31 a 50 anos & 63 & 31,50 \\
\hline Acima de 50 anos & 90 & 45,00 \\
\hline Gênero & & \\
\hline Masculino & 52 & 26,00 \\
\hline Feminino & 148 & 74,00 \\
\hline Estado Civil & & \\
\hline Solteiro & 32 & 16,00 \\
\hline Casado & 136 & 68,00 \\
\hline Outro & 32 & 16,00 \\
\hline Ocupação & & \\
\hline Desempregado & 36 & 18,00 \\
\hline Funcionário público & 4 & 2,00 \\
\hline Autônomo & 36 & 18,00 \\
\hline Assalariado & 49 & 24,50 \\
\hline Beneficiário & 75 & 37,50 \\
\hline Escolaridade & & \\
\hline Ensino fundamental & 76 & 38,00 \\
\hline Ensino médio & 109 & 54,50 \\
\hline Ensino superior & 8 & 4,00 \\
\hline Analfabeto & 7 & 3,50 \\
\hline Plano de Saúde Privado & & \\
\hline $\operatorname{Sim}$ & 12 & 6,00 \\
\hline Não & 188 & 94,00 \\
\hline
\end{tabular}

Fonte: elaboração própria (2018) 
Para o ano de 2019 , cerca de $34,44 \%$ das despesas do município foram alocadas para a saúde (SEBRAE, 2020). O município destaca-se regionalmente em determinadas especialidades tais como oncologia, neurologia, nefrologia e traumatologia. Diante de seu relatório de saúde atualizado em 2018 tem buscado direcionar suas ações voltadas a alimentação saudável, grupos de tabagismo e doenças respiratórias. Conta com 646 profissionais atuantes pelo SUS (FUMSSAR, 2018).

Em relação ao vínculo temporal existente entre usuário e sistema de saúde, $79,40 \%(n=158)$ dos participantes tem mais de 10 anos de vinculação ao serviço municipal de saúde de Santa Rosa/RS, $37,50 \%(n=75)$ frequentou a ESF de 1 a 3 vezes no último ano e $57,50 \%(n=115)$ recebeu de 1 a 3 vezes visita domiciliar de profissional da ESF no último ano, tal como evidenciado na Tabela 2.

Tabela 2 - Variáveis temporais dos participantes da pesquisa, Santa Rosa/RS, 2018

\begin{tabular}{|c|c|c|}
\hline \multirow{2}{*}{ Variável } & \multicolumn{2}{|c|}{$\mathrm{n}=\mathbf{2 0 0}$} \\
\hline & $\mathbf{n}$ & $\%$ \\
\hline \multicolumn{3}{|c|}{ Tempo de Vinculação com serviço de saúde municipal ${ }^{*}$} \\
\hline 1 a 3 anos & 17 & 8,54 \\
\hline 4 a 6 anos & 15 & 7,54 \\
\hline 7 a 10 anos & 9 & 4,54 \\
\hline mais de 10 anos & 158 & 79,40 \\
\hline \multicolumn{3}{|c|}{ Quantas vezes frequentou a ESF no último ano } \\
\hline 1 a 3 vezes & 75 & 37,50 \\
\hline 4 a 6 vezes & 47 & 23,50 \\
\hline 7 a 10 vezes & 19 & 9,50 \\
\hline mais de 10 vezes & 59 & 29,50 \\
\hline \multicolumn{3}{|c|}{$\begin{array}{l}\text { (Quantas vezes recebeu visita domiciliar de profissional da ESF no } \\
\text { último ano }\end{array}$} \\
\hline 1 a 3 vezes & 115 & 57,50 \\
\hline 4 a 6 vezes & 20 & 10,00 \\
\hline 7 a 10 vezes & 8 & 4,00 \\
\hline mais de 10 vezes & 57 & 28,50 \\
\hline
\end{tabular}

Fonte: elaboração própria (2018)

Quanto ao escore longitudinalidade, segundo o estudo, obteve-se um baixo grau de orientação à APS $(51,52 \%, n=102)$. O resultado foi avaliado como confiável, segundo o resultado do Alfa de Crombach $(0,743)$, considerando-se o valor de 0,70, tal como orientado por Hair Jr., Tatham e Black (2009). 
Tabela 3 - Associação das características socioeconômicas e demográficas com o alto/baixo escore da longitudinalidade no município de Santa Rosa/RS. Santa Rosa/RS, Brasil, 2018. $(n=198)$.

\begin{tabular}{|c|c|c|c|c|c|}
\hline \multirow{3}{*}{ Variáveis } & \multicolumn{5}{|c|}{ Longitudinalidade } \\
\hline & \multicolumn{2}{|c|}{$\begin{array}{c}\text { Baixo Escore } \\
(<6,6)\end{array}$} & \multicolumn{2}{|c|}{$\begin{array}{c}\text { Alto Escore } \\
(\geq 6,6)\end{array}$} & \multirow{2}{*}{$\begin{array}{c}\text { p- } \\
\text { valor* }\end{array}$} \\
\hline & $\mathbf{n}$ & $\%$ & $\mathbf{n}$ & $\%$ & \\
\hline \multicolumn{6}{|l|}{ Faixa Etária } \\
\hline Até 30 anos & 22 & 46,81 & 25 & 53,19 & \multirow{3}{*}{0,4415} \\
\hline 31 a 50 anos & 36 & 58,06 & 26 & 41,94 & \\
\hline Acima de 50 anos & 44 & 49,44 & 45 & 50,56 & \\
\hline Gênero & & & & & \multirow{3}{*}{0,3668} \\
\hline Masculino & 23 & 46,00 & 27 & 54,00 & \\
\hline Feminino & 79 & 53,38 & 69 & 46,62 & \\
\hline \multicolumn{6}{|l|}{ Estado Civil } \\
\hline Solteiro & 12 & 37,50 & 20 & 62,50 & \multirow{3}{*}{0,0356} \\
\hline Casado & 78 & 57,78 & 57 & 42,22 & \\
\hline Outro & 12 & 37,50 & 20 & 62,50 & \\
\hline \multicolumn{6}{|l|}{ Ocupação } \\
\hline Desempregado & 20 & 55,56 & 16 & 44,44 & \multirow{5}{*}{0,7083} \\
\hline Funcionário público & 2 & 66,67 & 1 & 33,33 & \\
\hline Autônomo & 20 & 57,14 & 15 & 42,86 & \\
\hline Assalariado & 26 & 53,06 & 23 & 46,94 & \\
\hline Beneficiário & 34 & 45,33 & 41 & 54,67 & \\
\hline \multicolumn{6}{|l|}{ Escolaridade } \\
\hline Ensino fundamental & 42 & 56,76 & 32 & 43,24 & \multirow{4}{*}{0,6099} \\
\hline Ensino médio & 54 & 49,54 & 55 & 50,46 & \\
\hline Ensino superior & 3 & 37,50 & 5 & 62,50 & \\
\hline Analfabeto & 3 & 42,86 & 4 & 57,14 & \\
\hline \multicolumn{6}{|l|}{ Plano de Saúde Privado } \\
\hline Sim & 6 & 54,53 & 5 & 45,45 & \multirow[t]{2}{*}{0,8361} \\
\hline Não & 96 & 51,34 & 91 & 48,66 & \\
\hline \multicolumn{6}{|c|}{ Tempo de Vinculação com serviço de saúde ${ }^{\star \star}$} \\
\hline 1 a 3 anos & 9 & 52,94 & 8 & 47,06 & \multirow{4}{*}{0,3630} \\
\hline 4 a 6 anos & 11 & 73,33 & 4 & 26,67 & \\
\hline 7 a 10 anos & 4 & 44,44 & 5 & 55,56 & \\
\hline mais de 10 anos & 78 & 50,00 & 78 & 50,00 & \\
\hline \multicolumn{6}{|c|}{ Quantas vezes frequentou a ESF no último ano } \\
\hline 1 a 3 vezes & 42 & 57,53 & 31 & 42,47 & \multirow{4}{*}{0,4229} \\
\hline 4 a 6 vezes & 23 & 48,94 & 24 & 51,06 & \\
\hline 7 a 10 vezes & 11 & 57,89 & 8 & 42,11 & \\
\hline mais de 10 vezes & 26 & 44,07 & 33 & 55,93 & \\
\hline \multicolumn{6}{|c|}{$\begin{array}{l}\text { Quantas vezes recebeu visita domiciliar de } \\
\text { profissional da ESF no último ano }\end{array}$} \\
\hline 1 a 3 vezes & 60 & 53,10 & 53 & 46,90 & \multirow{4}{*}{0,0380} \\
\hline 4 a 6 vezes & 14 & 70,00 & 6 & 30,00 & \\
\hline 7 a 10 vezes & 6 & 75,00 & 2 & 25,00 & \\
\hline mais de 10 vezes & 22 & 38,60 & 35 & 61,40 & \\
\hline
\end{tabular}

Para os escores relacionados à longitudinalidade em relação à classificação de baixa e alta longitudinalidade apresentaram associação, nas questões referentes ao estado civil e quantas vezes recebeu visita domiciliar $(p<0,05)$. Para uma análise mais abrangente relacionando todos os questionamentos com a longitudinalidade aplicou-se uma análise de clusters (Figura 1) para verificar quais variáveis se aproximam e a partir da aproximação, também confirmando as relações reclassificando as mesmas, utilizando análise de correspondência (Figura 2). 
Figura 1 - Análise de cluster entre as questões

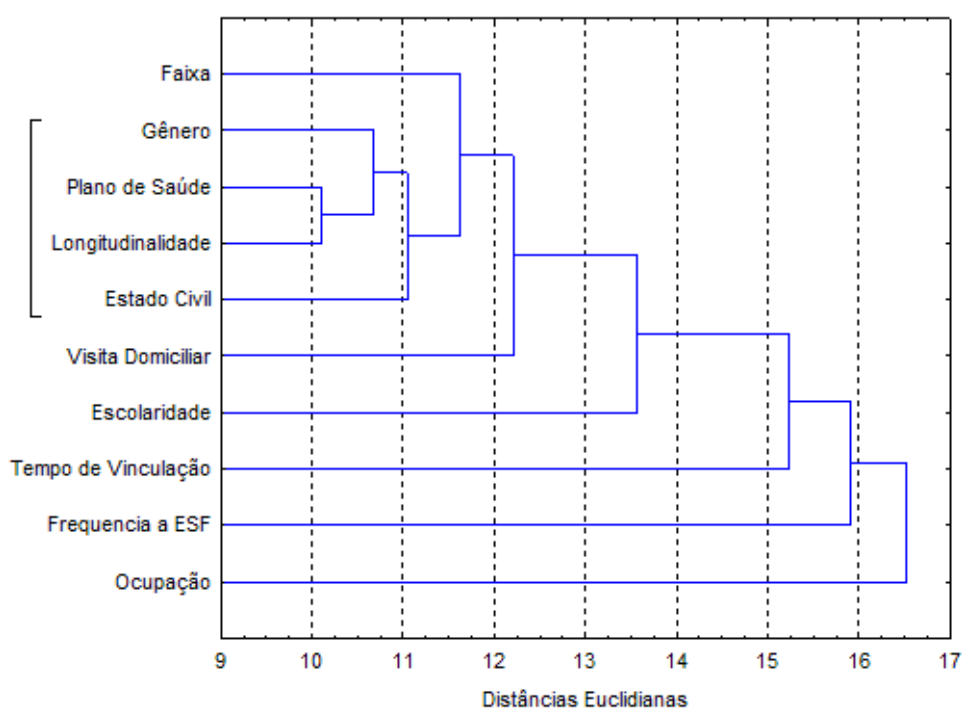

Fonte: elaboração própria (2018)

No dendograma da Figura 1, analisou-se na escala horizontal as similaridades entre as variáveis e na escala vertical estão listadas as variáveis em análise. As variáveis que mais se aproximam da dimensão longitudinalidade estão Plano de saúde, seguindo do gênero e estado civil. Relações semelhantes quanto à adesão aos planos de saúde privado também foram apresentadas por Fontenelle (2017), Tatangelo et al. (2017) e Dietrich, Colet e Winkelmann (2019). Na Figura 2 apresenta-se a relação espacial entre longitudinalidade e as variáveis mais próximas pela distância euclidiana.

Figura 2 - Análise de correspondência

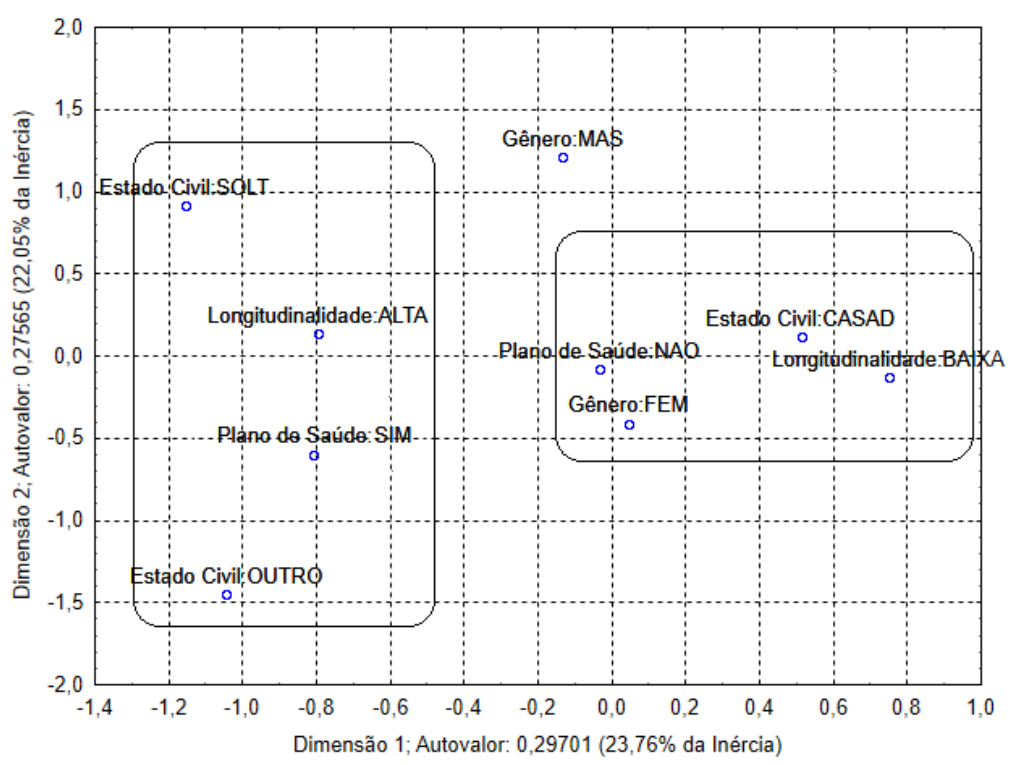

Fonte: elaboração própria (2018) 
Conforme apresentado na Figura 2, a análise de correspondência demonstrou que o plano de saúde privado não se relaciona com a longitudinalidade, em que também se verificou que, ter ou não ter plano de saúde é indiferente para os respondentes, visto que a maioria não possui planos de saúde e são usuários dos postos de saúde.

Esta análise retrata a realidade local dos usuários no munícipio de Santa Rosa/RS. Tais dados corroboram com a realidade a nível nacional, visto que cerca de $70 \%$ dos brasileiros não possuem plano de saúde privado e dependem exclusivamente do SUS (BOCCHINI, 2018). No que se refere ao gênero feminino, a longitudinalidade foi considerada baixa em destaque para a ausência de plano de saúde privado, no entanto para o gênero masculino, a longitudinalidade foi considerada alta, conforme pode ser observado nos pontos no espaço das distâncias euclidianas, em que prevaleceu a adesão ao plano de saúde privado, e estado civil solteiro.

Resultados diferentes foram encontrados por Dietrich, Colet e Winkelmann (2019) no munício de ljuí, também no estado do Rio Grande do Sul, em que as autoras encontraram maior incidência de adesão a planos privados de saúde por parte do gênero feminino e com alta taxa de escolaridade. Apesar de maior incidência de mulheres participantes nesta pesquisa e que condiz com a realidade local, existe uma predominância a nível nacional de os planos de saúde privados ser aderidos por elas (FONTENELLE, 2017). Diante de tal realidade, verificou-se neste estudo uma mudança em relação ao perfil comportamental na adesão aos planos de saúde privados, bem como ao estado civil, visto que a maior aproximação longitudinalidade foi verificada para homens solteiros. Neste sentido faz-se pertinente mencionar a baixa amostra de participantes que possuíam plano de saúde privado, $6 \%$ dos participantes.

\section{DISCUSSÃO}

As características socioeconômicas e demográficas que contemplam o perfil dos participantes deste estudo revelaram maior acesso ao sistema de saúde para pessoas com mais de 50 anos, em que predominou-se o gênero feminino (74\%). Os dados corroboram com os resultados encontrados em outros estudos desenvolvidos no país, os quais expressam que as mulheres possuem maior tendência pela procura de serviços de saúde, especialmente pelo SUS (HARZHEIM et al., 2013; OLIVEIRA, et al., 2013; KESSLER et al., 2019).

A maior incidência de cuidado da saúde por parte das mulheres também foi auferida por Levorato et al. (2014). Para os autores em questão, as mulheres possuem maior atenção ao cuidado à saúde em relação ao gênero masculino.

Em síntese, os resultados da pesquisa podem ser justificados devido à população santa-rosense ser predominantemente feminina, ademais a busca pelo sistema de saúde geralmente dá-se por parte destas, o que condiz com o panorama nacional pela busca de serviços de saúde, seja público ou privado.

A maioria dos/as participantes (68\%) é casado/a, o que corrobora com os achados do estudo de Araújo et al. (2014), em que $56 \%$ encontravam-se neste estado civil. Relativo à escolaridade ensino médio (54,5\%), também evidenciado no estudo de Harzheim et al. (2013) em Porto Alegre/RS, realizado com 2.404 usuários adultos, em que a escolaridade média foi de 9,6 anos de estudo e Araújo et al. (2014), realizado em Macaíba/RN com 100 usuários idosos, em que 58\% tinham algum grau de instrução.

Resultados semelhantes no que tange a este estudo, foram encontrados por Dietrich, Colet e Winkelmann (2019) no munício de ljuí/RS. Salienta-se neste sentido, que o maior percentual relativo à escolaridade ensino médio por parte dos participantes deste estudo está em concordância com o panorama de escolaridade apresentado no município de Santa de Rosa/RS, conforme dados demográficos da população.

Embora o direito à saúde esteja assegurado pela Constituição Federal Brasileira de 1988, o acesso nem sempre ocorre de maneira efetiva por parte dos Estados, assim uma das saídas encontradas por parte da população é a busca por planos privados de saúde (GRANDO; LUCION, 2016).

Relativo a este aspecto, identificou-se que a maioria dos participantes não possui (94\%) plano de saúde privado, o que corrobora com os achados de Kessler et al. (2019), em seu estudo com 1.067 usuários adultos, em que $74,13 \%$ não possuíam plano de saúde privado e evidenciado na Pesquisa 
Nacional de Saúde (PNS) 2013, em que somente cerca de 36,6\% da população do Rio Grande do Sul/RS possui plano de saúde privado.

A não adesão aos planos de saúde privados faz parte da realidade social brasileira, principalmente no que concerne a situação de pessoas em vulnerabilidade social e econômica, que por vezes por não possuir uma renda fixa acaba por depender exclusivamente dos serviços do SUS. Sob este viés, ressalta-se que a nível nacional com base nos dados do Instituto Brasileiro de Geografia e Estatística (IBGE) considerando-se o ano de 2019, estimou-se que mais de $70 \%$ dos brasileiros dependiam exclusivamente do SUS (MINISTÉRIO DA SAÚDE, 2021).

Apesar de parte da amostra deste estudo encontrar-se em situação de empregabilidade (24\%), observou-se que parte dos participantes encontrava-se em situação de desemprego (18\%) e trabalho autônomo (18\%), o que totaliza $36 \%$ dos participantes, o que explica tal realidade a nível municipal e a maior tendência na busca pelo SUS como sistema exclusivo para cuidar de sua saúde, e que também está alinhado ao panorama nacional brasileiro.

A longitudinalidade mais alta para homens, solteiros e que possuem plano de saúde privado demonstraram um perfil comportamental diferente do panorama nacional apresentado e que reflete de certa forma a uma mudança para este gênero no que tange a busca por cuidados e saúde no município objeto de estudo.

Neste sentido, salienta-se que sejam realizados maiores esforços para a promoção da saúde do homem por parte do sistema público, visto que geralmente estes não reconhecem "a atenção primaria como porta de entrada do sistema de saúde" (ALVES, et al, 2020, p.1). Ademais, salienta-se que os homens possuem uma expectativa de vida menor em relação às mulheres, justamente por não estarem atentos ao tratamento preventivo (CAMPOS, 2020).

Diante desta realidade, visto a parcela significativa de homens neste estudo, $26 \%$, e que apesar de inferior a participação feminina (74\%), demonstra-se um público-alvo que carece de atenção no que tange aos cuidados relativos à saúde, e principalmente na promoção por sua adesão e maior vínculo com o sistema de saúde.

Para que as estratégias da ESF sejam efetivas faz-se necessário o acompanhamento constante ao usuário (MINISTÉRIO DA SAÚDE, 2017). Os resultados demonstraram que a maioria dos participantes da pesquisa possui um tempo de vinculação considerado de longo prazo com os serviços de saúde do município, mais de dez anos, e que aufere um aspecto importante. Ainda, a maior parte destes, considerando-se o ano de 2018, frequentou a ESF de 1 a 3 vezes (37,\%), o que certifica um percentual interessante, mas que pode servir para se pensar novas estratégias na manutenção do vínculo e cuidado relativos à saúde por parte da gestão municipal. Notam-se os esforços das ESF nas visitas domiciliares, o que carece de disponibilidade de profissionais da saúde disponíveis, sendo que para este fator obteve-se um percentual significativo, e que indica o recebimento de visita domiciliar de 1 a 3 vezes (57,5\%), seguidos de mais de 10 vezes $(28,5 \%)$. Salienta-se neste contexto que o número de visitas também pode estar relacionado com as especificidades que envolvem a saúde de determinado paciente, e para tal, por vezes carecem de maior atenção e consequentemente maior número de visitas domiciliares por parte destes profissionais.

Diante dos achados a nível local, ressalta-se que no estado do Rio Grande do Sul/RS cerca de 47,8\% dos domicílios estão cadastrados em uma ESF há um ano ou mais e recebem visita domiciliar de algum profissional da ESF mensalmente. Ainda, $75,2 \%$ dos gaúchos tiveram ao menos uma consulta médica nos últimos 12 meses (IBGE, 2015).

Assim como as pesquisas realizadas anteriormente tanto em municípios do estado do Rio Grande do Sul, quanto de outros estados brasileiros, percebeu-se neste estudo um perfil demográfico predominante no acesso à saúde nas ESF, e que corresponde prioritariamente a mulheres, casadas e com ensino médio, o que possibilita pensar em estratégias para manutenção do vínculo existente.

A universalidade, a equidade e a integralidade são diretrizes básicas e essenciais nos serviços prestados na APS, o que perfaz desta forma a qualidade dos serviços (MINISTÉRIO DA SAÚDE, 2017). Relativo à satisfação quanto aos serviços prestados pelas EFS, com base na avaliação dos usuários participantes neste estudo e que possui relação à longitudinalidade, verificou-se, de modo geral, que os usuários avaliaram o serviço como insatisfatório e frágil, diante do baixo escore $(51,52 \%)$ em relação à longitudinalidade. 
Resultados semelhantes foram evidenciados no estudo de Vitoria et al. (2013) em Chapecó/SC, por meio de uma pesquisa realizada com médicos e enfermeiros da APS, que revelou um baixo escore para a longitudinalidade e também por Kessler et al. (2019), com 1.076 adultos que demonstrou presença insatisfatória da longitudinalidade. Ainda, a insatisfação quanto aos serviços das ESF também foi encontrada no estudo de Moysés et al. (2019), realizado com 857 mulheres de três municípios da Amazônia.

O estudo de Harmuch e Baratieri (2017), realizado com 379 usuários no município de Guarapuava/PR, no período de 2015 e 2016, também apresentou uma avaliação negativa da APS, já Kessler et al. (2019), evidenciou melhores avaliações somente em municípios de menor porte populacional, levando-se em consideração a avaliação de 1.076 usuários de 32 munícipio da $4^{\text {a }}$ Coordenadoria Regional de Saúde do Rio Grande do Sul/RS, considerando-se o ano de 2015.

Um dos motivos da ocorrência do escore diminuído para a longitudinalidade pode estar associado à acessibilidade prejudicada nos serviços da APS (SILVA et al., 2013) e à inexistência de perfil dos trabalhadores de saúde para a atuação na APS (ARAÚJO et al., 2011).

Neste sentido, um grande obstáculo a ser enfrentado para o alcance da longitudinalidade de maneira plena é a má formação e qualificação profissional, que reflete em uma assistência à saúde segmentada e desassociada da real vida e condição de saúde da população adstrita ao serviço de saúde (OLIVEIRA; PEREIRAM 2013).

Para a efetividade dos atendimentos alguns aspectos como conhecer o histórico clínico e os antecedentes dos pacientes faz o profissional de saúde atingir uma análise mais exata de sua situação (STARFIEDLD, 2002). Isso permite ao profissional planejar de maneira mais eficiente e assertiva as intervenções e o cuidado a ser realizado ao paciente (BARATIERI; MANDU; MARCON, 2012).

O baixo escore para a longitudinalidade na APS por meio de sua insatisfação indica uma possível tendência à desistência do usuário ao serviço público de saúde (FRANK et al., 2015). Diante desta realidade em específico, fazem-se necessárias estratégias voltadas ao fortalecimento e a reorganização da atenção à saúde (VALE et al., 2019).

Este estudo demonstrou a fragilidade na construção do vínculo ao longo do tempo entre os usuários do sistema de saúde e os profissionais que atuam nas ESF's no município de Santa Rosa, fator este que pode direcionar ao afastamento do usuário caso não sejam implementadas estratégias voltadas a saúde, ao cuidado e a manutenção desses vínculos. Ainda, diante da baixa participação do público masculino, ressalta-se a necessidade de ações a serem promovidas especificamente para estes. Apesar do tempo existente de vínculo ser superior a dez anos, e a frequência de visitas a ESF ser de 1 a 3 vezes por ano, faz-se necessário atentar ao perfil predominante neste estudo, trata-se de pessoas com mais de 50 anos (45\%), o que de certa forma requer maior atenção por parte dos profissionais da saúde.

Os resultados apresentados neste estudo e que evidenciaram a realidade local do município em estudo, demonstraram a necessidade de um olhar mais atento por parte de gestores e profissionais da saúde empenhados pela prestação dos serviços pelas ESF. Assim, fazem-se necessárias novas estratégias que permitam atentar a longitudinalidade e melhor atendimento aos usuários.

\section{CONSIDERAÇÕES FINAIS}

A avaliação da longitudinalidade do cuidado pela perspectiva dos usuários no município de Santa Rosa/RS permitiu identificar a insatisfação quanto a este atributo no serviço de saúde prestado e nas relações estabelecidas pelas ESF.

Diante desta realidade, ressalta-se a importância da reestruturação dos serviços de saúde, juntamente com a sensibilização dos profissionais que atuam nos serviços e que estão em contato próximo com o paciente, com o objetivo de reflexão da prática profissional, fortalecimento do vínculo e melhor desempenho nas ações realizadas rotineiramente nas unidades de saúde.

A capacitação e presença da educação permanente devem ser estimuladas entre os gestores de saúde para a qualificação dos serviços prestados à população, com o objetivo de fomentar o cuidado integral ao paciente, levando em conta não só aspectos biológicos, mas sim sua cultura e o meio em que o usuário está inserido. 
Como limitações do estudo, destaca-se a fragilidade da amostra, composta somente por usuários que frequentaram a unidade na data da coleta de dados. Em relação aos resultados apresentados em outras pesquisas, salientam-se os diferentes contextos geográficos do país, e que refletem consequentemente em características específicas no que tange ao perfil sócio demográfico.

Esse estudo oferece aporte e serve de parâmetro para gestores, pesquisadores e profissionais de saúde, para que possam embasar suas práticas de gestão e financiamento, com o objetivo de qualificar e aproximar os profissionais das ESF aos usuários e para que o serviço de saúde forneça maior resolutividade e melhor construção de vínculo profissional/gestão com o usuário de saúde.

Sugere-se que sejam realizados estudos voltados a avaliação da longitudinalidade por parte dos usuários anualmente no município de Santa Rosa/RS, e em outros municípios da região noroeste do estado do Rio Grande do Sul, e posterior comparativo sobre o atendimento à saúde a nível regional. Ainda, que sejam realizados estudos qualitativos voltados a compreensão dos aspectos que geram sua satisfação e insatisfação quanto aos atendimentos com base na opinião dos usuários. Salienta-se também a possibilidade de pesquisas qualitativas voltadas a percepção de gestores/as e enfermeiros/as para melhor entendimento das práticas que vem sendo realizadas em prol da APS e da longitudinalidade no município de Santa Rosa/RS.

\section{REFERÊNCIAS}

ALVES, M.T.G. Reflexões sobre o papel da Atenção Primária à Saúde na pandemia de COVID-19. Revista Brasileira de Medicina de Família e Comunidade. v. 15, n. 42, 2020. https://doi.org/10.5712/rbmfc15(42)2496

ALVES, A.N. et al. Acesso de primeiro contato na atenção primária: uma avaliação pela população masculina. Revista Brasileira de Epidemiologia, 2020. https://doi.org/10.1590/1980-549720200072

ARAÚJO, R. M. A.; MARTINS, T. C. P.; COTTA, R. M. M.; CHERCHIGLIA; M. L. Atenção Primária à Saúde a "menina dos olhos" do SUS: sobre as representações sociais dos protagonistas do Sistema Único de Saúde. Ciência e Saúde Coletiva. v. 16, p. 881-89, 2011. https://doi.org/10.1590/S1413$\underline{81232011000700020}$

ARAÚJO, L. U. A.; OliVEIRA, F. L. A.; AZEVEDO, W. M.; ALMEIDA Jr., H. J. B. Avaliação da qualidade da atenção primária à saúde sob a perspectiva do idoso. Ciência \& Saúde Coletiva. v. 19, n. 8, p. 3521-3532, 2014. https://doi.org/10.1590/1413-81232014198.21862013

BARATIERI, T.; MANDU, E. N. T.; MARCON, S. S. Longitudinalidade no trabalho do enfermeiro: relatos da experiência profissional. Revista da Escola de Enfermagem da USP. v. 6, n. 5, p. 12601267, 2012. https://doi.org/10.1590/S0080-62342012000500031

BOCCHINI, B. Pesquisa mostra que quase $70 \%$ dos brasileiros não têm plano de saúde particular. 2018. Disponível em <https://agenciabrasil.ebc.com.br/geral/noticia/2018-02/pesquisamostra-que-quase-70-dos-brasileiros-nao-tem-plano-de-saude-particular>. Acesso em 11 de julho de 2021.

BRASIL. Ministério da Saúde. Programa Saúde da Família. Brasília, 2004.

Ministério da Saúde. Secretaria de Atenção em Saúde. Departamento de Atenção Básica. Manual do Instrumento de Avaliação da Atenção Primária à Saúde. Brasília, 2010.

Portaria no. 2.488 de 21 de outubro de 2011. Aprova a Política Nacional de Atenção Básica, estabelecendo a revisão de diretrizes e normas para organização da Atenção Básica para o Programa Saúde da Família (PSF) e o Programa Agentes Comunitários de Saúde (PACS), 2011.

Conselho Nacional de Saúde. Resolução №466/2012 de 12 de dezembro de 2012. Trata das Diretrizes e Normas Regulamentadoras de Pesquisa envolvendo Seres Humanos. Brasília: Ministério da Saúde, 2012.

CABRAL, K.F.D.; FERREIRA, M. A. M.; BATISTA, R. S.; CARQUEIRA, F. R. Atenção primária à saúde: uma análise a luz da eficiência técnica dos recursos no Estado de Minas Gerais. Revista de Gestão e Sistemas de Saúde. São Paulo, v. 8, n. 2, p. 137-150, 2019. https://doi.org/10.5585/rgss.v8i2.14923 
CAMPOS, A. C. IBGE: esperança de vida do brasileiro aumentou 31,1 anos desde 1940. 2020. Disponível em:< https://agenciabrasil.ebc.com.br/geral/noticia/2020-11/ibge-esperanca-de-vida-dobrasileiro-aumentou-311-anos-desde-1940>. Acesso em 11 de julho de 2021.

CARVALHO, V. C. H. S.; ROSSATO, S. L.; FUCHS, F. D.; HARZHEIM, E.; FUCHS, S. C. Assessment of primary health care received by the elderly and health related quality of life: a cross-sectionalstudy. BMC Public Health. v. 13, n. 605, p. 1-9, 2013. https://doi.org/10.1186/1471-2458-13-605

CASTRO, S. S. C.; ANES, C. E. R.; DALCIN, F. O planejamento da fundação municipal de saúde de SANTA ROSA/RS: diretrizes para seu desenvolvimento. Desenvolvimento Regional: Processos, Políticas e Transformações Territoriais. Santa Cruz do Sul/RS, 2019.

CUNHA, E. M.; GIOVANELLA, L. Longitudinalidade/continuidade do cuidado: identificando dimensões e variáveis para a avaliação da Atenção Primária no contexto do sistema público de saúde brasileiro. Ciência \& Saúde Coletiva. v. 16, p. 1029-1042, 2011. https://doi.org/10.1590/S1413$\underline{81232011000700036}$

DAMACENO, A.N.; LIMA, M. A. D. S.; PUCCI, V. R.; WEILLER, T. H. Redes de atenção à saúde: uma estratégia para integração dos sistemas de saúde. Revista de Enfermagem da UFSM. v. 10, p. 1-14, 2020. https://doi.org/10.5902/2179769236832

DUNCAN, B. B.; SCHMIDT, M. I.; GIUGLIANI, E. R. J. Medicina ambulatorial:Condutas de Atenção Primária Baseadas em Evidências. Porto Alegre: Artmed, 4. ed, 2004.

DIETRICH, A.; COLET, C. F.; WINKELMANN, E. R. Perfil de saúde dos usuários da rede de atenção básica baseado no cadastro individual e-Sus. Revista de Pesquisa. v.1 1, n. 5, p. 1266-1271, 2019. https://doi.org/10.9789/2175-5361.2019.v1115.1266-1271

DUTRA, E. B., GOMES, V. C. Qualidade da saúde no Brasil: um retrato do seu desempenho baseado na abordagem sistêmica. Revista de Gestão e Sistemas de Saúde. São Paulo, v. 9, n. 3, p. 554577, 2020. https://doi.org/10.5585/rgss.v9i3.17257

FONTENELLE, L.F. Nível socioeconômico, cobertura por plano de saúde, e autoexclusão do Sistema Único de Saúde. 157f. Tese (Programa de Pós-graduação em Epidemiologia). Universidade Federal de Pelotas, 2017.

FRANK, B. R. B.; VIEIRA, C. S. ; ROSS, C. ; OBREGÓN, P. L.; TOSO, B. R. G. O. Avaliação da longitudinalidade em unidades de Atenção Primária à Saúde. Saúde em Debate. v. 39, n. 105, p. 400-410, 2015. https://doi.org/10.1590/0103-110420151050002008

FUNDAÇÃO MUNICIPAL DE SAÚDE (FUMSSAR). Relatório detalhado do quadrimestre anterior RDQA período de setembro a dezembro - 3ำ quadrimestre de 2018 e relatório anual de saúde 2018, 2018.

GÉRVAS, J.; FERNÁNDEZ, M. P. El fundamento científico de lafunción de filtro del médico general. Revista Brasileira de Epidemiologia. v. 8, n. 2, p. 205-218, 2005. https://doi.org/10.1590/S1415790X2005000200013

GÉRVAS, J.; FERNÁNDEZ, M. P.; SÁNCHEZ, R. J. S. Longitudinalidad, prestigio, buenareputación (social y profesional) y medicina general/de familia. Aspectos clínicos y de salud pública. Gaceta Sanitaria. v. 26, p. 52-56, 2012. https://doi.org/10.1016/i.gaceta.2011.09.024

GIOVANELLA, L.; BOUSQUAT, A. E. M.; SCHEKMAN, S.; ALMEIDA, P. F.; SARDINHA, L. M. F. P. Cobertura da Estratégia Saúde da Família no Brasil: o que nos mostram as Pesquisas Nacionais de Saúde 2013 e 2019. Ciência \& Saúde Coletiva. v. 26, p. 2543-2556, 2021. https://doi.org/10.1590/1413-81232021266.1.43952020

GRANDO, J. B.; LUCION, M.C. S. O direito fundamental à saúde e (des)respeito dos planos de saúde. Direito em Debate - Revista do Departamento de Ciências Jurídicas e Sociais da Uniju. v. 25, n. 46, p. 3-22, 2016. https://doi.org/10.21527/2176-6622.2016.46.3-22

HAIR Jr., J. F.; BABIN, B.; MONEY, A.H.; SAMOUEL, P. Fundamentos de métodos de pesquisa em administração. Tradução Lene Belon Ribeiro. Porto Alegre: Bookman, 2005. 471p.

HAIR Jr., A. R. E.; TATHAM, R. L.; BLACK, W. C. Análise Multivariada de Dados. 5. ed. Porto Alegre: Bookman, 2009. 
HARMUCH, C.; BARATIERI, T. Avaliação da longitudinalidade na atenção primária à saúde sob a ótica dos usuários. Revista Varia Scientia - Ciências da Saúde. v. 3, n. 1, p. 16-25, 2017.

HARZHEIM, E.; STARFIELD, B.; RAJMIL, L.; ÁLVAREZ-DARDET, C.; STEIN, A. T. Consistência interna e confiabilidade da versão em português do Instrumento de Avaliação da Atenção Primária (PCATool-Brasil) para serviços de saúde infantil. Cadernos de Saúde Pública. Rio de Janeiro, v. 22, n. 8, p. 1649-1659, 2006. https://doi.org/10.1590/S0102-311X2006000800013

HARZHEIM, E.; OLIVEIRA, M. M. C.; AGOSTINHO, M. R.; HAUSER, L.; STEIN, A. T.; GONÇALVES, M. R.; TRINDADE, T. G.; DUNCAN, B. B.; STARFIELD, B.Validação do instrumento de avaliação da atenção primária à saúde: PCATool-Brasil adultos. Revista Brasileira de Medicina de Família e Comunidade. v.8, n.29, p. 274-84, 2013. https://doi.org/10.5712/rbmfc8(29)829

HARZHEIM, E.; SORANZ, D.; HAUSR, L.; PINTO, L. F. Avaliação dos usuários crianças e adultos quanto ao grau de orientação para Atenção Primária à Saúde na cidade do Rio de Janeiro, Brasil. Ciência \& Saúde Coletiva. v.21, n.5, p. 1399-1408, 2016. https://doi.org/10.1590/1413$\underline{81232015215.26672015}$

HARZHEIM, E.; SANTOS, C. M. J.; D'AVILA, O. P.; WOLMANN, L.; PINTO, L. F. Bases para a reforma da Atenção Primária à Saúde no Brasil em 2019: mudanças estruturantes após 25 anos do Programa de Saúde da Família. Revista Brasileira de Medicina de Família. Rio de Janeiro, v.15, n.42, p.1-16, 2020. https://doi.org/10.5712/rbmfc15(42)2354

INSTITUTO BRASILEIRO DE GEOGRAFIA E ESTATíSTICA (IBGE). Pesquisa Nacional de Saúde 2013: acesso e utilização dos serviços de saúde, acidentes e violências: Brasil, grandes regiões e unidades da federação. Rio de Janeiro, 2015.

.Estimativas de População no DOU, 2017.

KESSLER, M.; LIMA, S. B. S.; WEILLER, T. H.; LOPES, L. F. D.; FERRAZ, L.; EBERHARDT, T. D.; SOARES, R. S. Á.; TRINDADE, L. L. Longitudinalidade do cuidado na atenção primária: avaliação na perspectiva dos usuários. Acta Paulista de Enfermagem. São Paulo, v. 32, n. 2, p. 186-193, 2019. https://doi.org/10.1590/1982-0194201900026

LAVRAS, C. Atenção Primária à saúde e a organização de redes regionais de atenção a saúde no Brasil. Saúde e Sociedade. v. 20, n.4, p. 867-874, 2011. https://doi.org/10.1590/S010412902011000400005

LEVORATO, C. D.; MELLO, L. M.; SILVA, A. S.; NUNES, A. A. Fatores associados à procura por serviços de saúde numa perspectiva relacional de gênero. Ciência Saúde Coletiva, v. 19, n. 4, 2014. https://doi.org/10.1590/1413-81232014194.01242013

MINISTÉRIO DA SAÚDE. Portaria no. 2.436, de 21 de setembro de 2017. Aprova a Política Nacional de Atenção Básica, estabelecendo a revisão de diretrizes para a organização da Atenção Básica, no âmbito do Sistema Único de Saúde (SUS), 2017.

Portaria no. 2.979, de 12 de novembro de 2019. Institui o Programa Previne Brasil, que estabelece novo modelo de financiamento de custeio da Atenção Primária à Saúde no âmbito do Sistema Único de Saúde, por meio da alteração da Portaria de Consolidação no 6/GM/MS, de 28 de setembro de 2017. 2019.

DiretrizesEstratégicas.

Disponível

em

$\overline{<h t t p s: / / b v s m s . s a u d e . g o v . b r / b v s / p a c s a u d e / d i r e t r i z e s . p h p>. ~ A c e s s o ~ e m ~} 09$ de julho de 2021.

MOYSÉS, R. P. C.; TEIXEIRA, V. C. L.; MARTINS, R. G.; SOUZA, C. S. M.; ABENSUR, T. C.; PREREIRA, M. G. Integralidade e longitudinalidade da Atenção Primária à Saúde da mulher: uma análise de três municípios amazônicos. Revista de APS. v. 22 , n. 1, p. 168-182, 2019. https://doi.org/10.34019/1809-8363.2019.v22.16740

OLIVEIRA, M.A.C.; PEREIRA, I.C. Atributos essenciais da Atenção Primária e a Estratégia Saúde da Família. Revista Brasileira de Enfermagem. v. 66, p. 158-164, 2013. https://doi.org/10.1590/S0034$\underline{71672013000700020}$

OLIVEIRA, M. M. C.; HARZHEIN, E.; RIBOLDI, J. DUNCAN, B. B. PCATool - Adulto - Brasil: uma versão reduzida. Revista Brasileira de Medicina de Família e Comunidade. v. 8, n.29, p. 256-263, 2013. https://doi.org/10.5712/rbmfc8(29)823

DOI: http://dx.doi.org/10.14393/Hygeia17059172

Hygeia

v.17

p. $301-317$,

2021 página 315 
RASIA, I. C. R. B.; SILVEIRA, D. S.; OLEA, P. M.; RASIA, B. B. Estruturação e orientação da atenção primária à saúde em um município no extremo sul do Brasil. Revista de Gestão e Sistemas de Saúde. São Paulo, v. 9, n. 2, p. 193-214, 2020. https://doi.org/10.5585/rgss.v9i2.14493

RODRIGUES, M. J.; RAMIRES, J. C. L. Estratégia de saúde da família: desafios para sua consolidação em Uberlândia. Revista Brasileira de Geografia Médica e da Saúde-HYGEIA. v. 11, n. 20, p. 153-166, 2015.

SANTOS, A.M. Redes regionalizadas de atenção à saúde desafios à integração assistencial e à coordenação do cuidado. Editora da Universidade Federal da Bahia, 2018. https://doi.org/10.7476/9788523220266

SANTOS, R. O. M.; ROMANO, V. F.; ENGSTROM, E. M. Vínculo longitudinal na Saúde da Família: construção fundamentada no modelo de atenção, práticas interpessoais e organização dos serviços. Physis: Revista de Saúde Coletiva. Rio de Janeiro, v. 28, n. 2, p. 1-18, 2018. https://doi.org/10.1590/s0103-73312018280206

SCHENKER, M.; COSTA, D. H. Avanços e desafios da atenção à saúde da população idosa com doenças crônicas na Atenção Primária à Saúde. Ciência e Saúde Coletiva. v. 24 , n. 4, p. 13691379, 2019. https://doi.org/10.1590/1413-81232018244.01222019

SEBRAE. Perfil das cidades gaúchas-Santa Rosa. DATABASE, 2020. Disponível em < http://datasebrae.com.br/municipios/rs/Perfil_Cidades_Gauchas-Santa_Rosa.pdf $>$. Acesso em 8 de julho de 2021.

SECRETARIA DE ATENÇÃO PRIMÁRIA À SAÚDE (SAPS). O que é Atenção Primária?. Disponível em: < https://aps.saude.gov.br/smp/smpoquee>. Acesso em 21 de novembro de 2020.

SECRETARIA DA SAÚDE. 14a CRS (Santa Rosa). Disponível em: < https://saude.rs.gov.br/14-crssanta-rosa>. Acesso em 08 de julho de 2021.

SILVA, R. M. M.; VIEIRA, C. S.; TOSO, B. R. G. O.; NEVES, E. T.; RODRIGUES, R. M. Resolutividade na atenção à saúde da criança: percepção de pais e cuidadores. Acta Paulista de Enfermagem. v. 26, n. 4, p. 382-388, 2013. https://doi.org/10.1590/S0103-21002013000400013

SILVEIRA, C. B.; JORGE, M. S. B.; LEITÃO, I. M. T. A. As redes de atenção à saúde e suas interfaces: processos de trabalho, atenção primária à saúde e produção do cuidado integral. In: JORGE, M.S.B.; BEZERRA, I.C.; LEITÂO, I.M.T.A. (Org). Pesquisas em saúde no contexto do cuidado, redes de atenção, fluxos e avaliação: multiplicidade de olhares. Fortaleza: EdUECE, p. 117, 2017.

SOUZA, M. F. M.; MALTA, D. C. M; FRANÇA, E.; BARRETO; M. Transição da saúde e da doença no Brasil e nas Unidades Federadas durante os 30 anos do Sistema Único de Saúde. Ciência e Saúde Coletiva. v. 23, n.6, p. 1737-1750, 2018. https://doi.org/10.1590/1413-81232018236.04822018

SOUZA, L. E. P. F.; PAIM, J. S.; TEIXEIRA, C. F.; BAHIA, L.; GUIMARÃES, R.; ALMEIDA-FILHO, N.; MACHADO, C. V.; CAMPOS, G. W.; AZEVEDO-E-SILVA, G. Os desafios atuais da luta pelo direito universal à saúde no Brasil. Ciência e Saúde Coletiva. v. 24, n. 8, p. 2783-2792, 2019. https://doi.org/10.1590/1413-81232018248.34462018

STARFIELD, B. Atenção primária: equilíbrio entre necessidades de saúde, serviços e tecnologia. Brasília: UNESCO, Ministério da Saúde, 2002.

STARFIELD, B.; SHI, L. Manual for the Primary Care Assessment Tools. Baltimore: Johns Hopkins University, 2002.

SZWARCWALD, C. L.; MONTILLA, D. E. R.; MARQUES, A. P.; DAMACENA, G. N.; ALMEIDA, W. S.; MALTA, D. C. Desigualdades na esperança de vida saudável por Unidades da Federação. Revista Saúde Pública [online]. v. 51, p.1-11, 2017. https://doi.org/10.1590/s1518-8787.2017051000105

TATANGELO, G.; McCABE, M.; CAMPBELL, S.; SZOEKE, C. Gender, marital status and longevity. Maturitas. v. 100, p. 64-69, 2017. https://doi.org/10.1016/j.maturitas.2017.03.002

TESSER, C. D.; NORMAN, A. H.; VIDAL, T. B. Acesso na Atenção Primária à Saúde brasileira: situação, problemas e estratégias de superação. Saúde Debate, v. 42, n. 1, p. 361-78, 2018. https://doi.org/10.1590/0103-11042018s125 
VALE, P.R.L.F.; PRATA, D. et al. Fortalecendo a longitudinalidade do cuidado aos sujeitos participantes do programa Hiperdia. Revista de APS. v. 22, n. 2, p. 479-490, 2019. https://doi.org/10.34019/1809-8363.2019.v22.15918

VITORIA, A. M.; HARZHEIM; E.; TAKEDA, S. P.; HAUSER, L. valiação dos atributos da atenção primária à saúde em Chapecó, Brasil. Revista Brasileira de Medicina de Família e Comunidade. v. 8, n. 29, p. 285-293, 2013. https://doi.org/10.5712/rbmfc8(29)832 